\title{
Structural shape sensitivity analysis for nonlinear material models with strain softening
}

\author{
G. Bugeda, L. Gil and E. Oñate
}

Escola Tècnica Superior d'Enginyers de Camins, Canals i Ports; Universitat Politècnica de Catalunya Campus Nord UPC, Mòdul C1, C/ Gran Capità s/n, 08034 Barcelona, Spain

\begin{abstract}
This paper describes some considerations around the analytical structural shape sensitivity analysis when the structural behaviour is computed using the finite element method with a nonlinear constitutive material model. Traditionally, the structural sensitivity analysis is computed using an incremental approach based on the incremental procedures for the solution of the structural equilibrium problem. In this work, a direct (nonincremental) formulation for computing these structural sensitivities, that is valid for some specific nonlinear material models, is proposed. The material models for which the presented approach is valid are characterized by the fact that the stresses at any time $t$ can be expressed in terms of the strains at the time $t$ and, in some cases, the strains at a specific past time $t^{2}\left(t^{u}<t\right)$. This is the case of elasticity (linear as well as nonlinear), perfect plasticity and damage models. A special strategy is also proposed for material models with strain softening.

For the cases where it is applicable, the sensitivity analysis proposed here allows us to compute the structural sensitivities around any structural equilibrium point after finishing the solution process and it is completely independent of the numerical scheme used to solve the structural equilibrium problem. This possibility is particularized for the case of a damage model considering a strain-softening behaviour. Finally, the quality and reliability of the proposed approach is assessed through its application to some examples.
\end{abstract}

\section{Introduction}

Roughly speaking, sensitivities are understood as a relation between some control parameters, such as the structural response, and some design variables that define the structural shape. From this point of view, if a design variable $q$ is selected, the sensitivity of the structural response can be defined in terms of displacements $\mathbf{u}$ as the relation $\mathrm{d} \mathbf{u} / \mathrm{d} q$ or as $\mathrm{d} \sigma / \mathrm{d} q$ in terms of stresses.

In optimization problems these sensitivities are normally defined in terms of the objective function and the restrictions. In general, these functions depend on the structural response and, due to that, in this work only the sensitivities in terms of the displacements or the stresses have been considered.

There have been different contributions on performing the sensitivity analysis in nonlinear structural systems (Choi and Santos 1987, 1992; Arora and Cardoso 1988, 1992; Tsay and Arora 1990; Tsay et al. 1990; Yao and Arora 1992a,b; Kleiber 1993). In particular, for the case of nonlinear material mod- els, most research is related to the use of plasticity models (see Haslinger and Mäkinen 1992; Vidal and Haber 1993; Kleiber et al. 1994, 1995; Kleiber and Hien 1996). Due to the fact that using an incremental approach solves most of the nonlinear structural equilibrium problems, the sensitivity analysis is normally obtained as an addition of increments

$\frac{t+\Delta t_{\mathrm{d}} \mathbf{u}}{\mathrm{d} q}=\frac{{ }^{t} \mathrm{~d} \mathbf{u}}{\mathrm{d} q}+\Delta\left(\frac{\Delta t_{\mathrm{d} \mathbf{u}}}{\mathrm{d} q}\right)$.

The incremental magnitude of the sensitivities are obtained at each step of the incremental approach through the differentiation of the incremental integral equilibrium equation (Vidal et al. 1993),

$\frac{\mathrm{d}}{\mathrm{d} q}\left[\int_{V} \mathbf{B}^{\mathrm{T}} \dot{\boldsymbol{\sigma}} \mathrm{d} V-\dot{\mathbf{f}}\right]=0$,

where $\mathbf{B}$ is the deformation matrix, $\boldsymbol{\sigma}$ is the increment vector and $\dot{\mathbf{f}}$ is the external forces increment vector.

Another alternative is to start from the incremental finite element discrete equilibrium equations (Kleiber et al. 1995, 1996)

$\mathbf{K}_{\mathrm{T}}(\mathbf{u}, q) \Delta \mathbf{u}(q)={ }^{t+\Delta t^{t}} \mathbf{f}-{ }^{t} \mathbf{r}$,

where $\mathbf{K}_{\mathrm{T}}$ is the tangent stiffness matrix, $\Delta \mathbf{u}$ is the incremental displacement vector, ${ }^{t+\Delta t_{\mathbf{f}}}$ the nodal external forces vector and ${ }^{t} \mathbf{r}$ is the nodal internal forces vector corresponding to the last equilibrium configuration.

These strategies involve high computational cost because the sensitivity analysis must be computed after the convergence of each load increment. In addition, the incremental nature makes it suitable to carry and to accumulate errors depending on the resolution strategy used to solve the equilibrium problems. Then, the quality of this type of sensitivity analysis can depend on the resolution strategy and the size of the load increments.

Another general approach of the sensitivity analysis with nonlinear material problems appears in the paper by Ryu et al. (1985), and is applied to plasticity by Silva et al. (1997). In this last reference the differentiation of the global equilibrium equation in its integral form is proposed,

$$
\frac{\mathrm{d}}{\mathrm{d} q}\left[\int_{V} \mathbf{B}^{\mathrm{T}} \boldsymbol{\sigma} \mathrm{d} V-\mathbf{f}\right]=0,
$$


where $\boldsymbol{\sigma}$ are stresses and $\mathbf{f}$ is the external nodal forces vector.

The present work follows similar arguments and a direct strategy for the evaluation of the sensitivities at the equilibrium points without any incremental dependence is proposed. In addition, in order to solve problems involving constitutive material models with strain softening a specific strategy is also proposed.

The following sections present the proposed sensitivity analysis formulation. 'Two different concepts are developed. The first one is a formulation for the sensitivity analysis of a nonlinear constitutive material model without taking into account, yet, the possibility of strain-softening behaviour. This concept is particularized for the case of a damage model. The second one is the possibility of considering a constitutive material model with strain softening. The additional considerations to take it into account are developed for the case of the damage model with strain softening and can be easily generalized for any other constitutive model. One of these additional considerations is the necessity of including a special approach based on an arc-length strategy for a proper use of the sensitivity analysis to project the structural behaviour from an original structure to a modified one. Finally, the use of all the formulations presented is illustrated through some assessment examples.

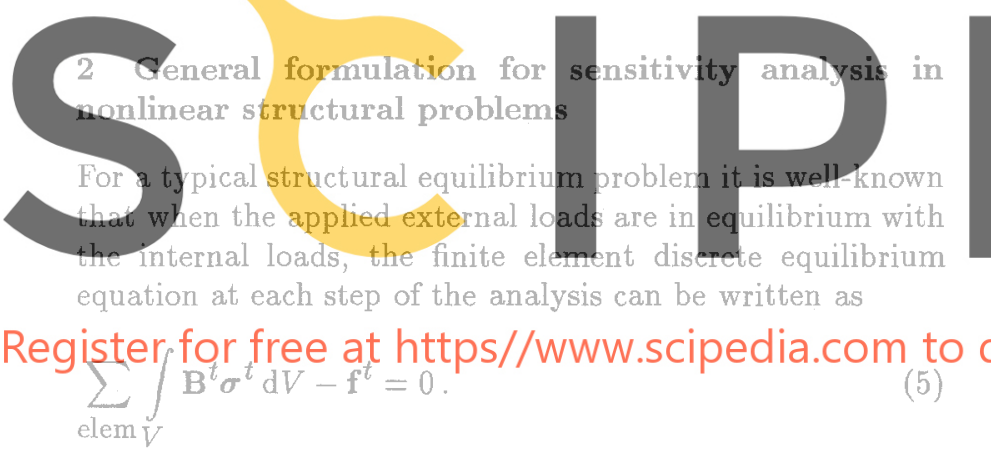

Here we have used the superscript $t$ to indicate the pseudotime that corresponds to the step of the analysis in which the equilibrium equation has been considered.

Taking into account that our objective is to compute the sensitivities at the equilibrium point we can differentiate the discrete equilibrium equation with respect to a design variable $q$,

$$
\begin{aligned}
& \frac{\mathrm{d}}{\mathrm{d} q}\left[\sum_{\text {elem }} \int_{V} \mathbf{B}^{\mathrm{T}} \boldsymbol{\sigma}^{t} \mathrm{~d} V-\mathbf{f}^{t}\right]= \\
& \sum_{\text {elem }}\left[\frac{\mathrm{d}}{\mathrm{d} q} \int_{V} \mathbf{B}^{\mathrm{T}} \boldsymbol{\sigma}^{t} \mathrm{~d} V\right]-\frac{\mathrm{d} \mathbf{f}^{t}}{\mathrm{~d} q}=0 .
\end{aligned}
$$

The last expression can be developed for each finite element of the mesh. Assuming that the iso-parametric transformation can be used for the integration domain of each element, the last expression becomes

$$
\sum_{\text {elem }}\left[\int_{V} \frac{\mathrm{d}}{\mathrm{d} q}\left(\mathbf{B}^{\mathrm{T}} \boldsymbol{\sigma}^{t}|\mathbf{J}|\right) \mathrm{d} V_{0}\right]-\frac{\mathrm{d} \mathbf{f}^{t}}{\mathrm{~d} q}=0,
$$

where $\mathbf{J}$ is the Jacobian of the iso-parametric transformation. If now we develop (7) for each element of the finite element mesh we obtain

$$
\begin{aligned}
& \int_{V_{0}} \frac{\mathrm{d}}{\mathrm{d} q}\left(\mathbf{B}^{\mathrm{T}} \boldsymbol{\sigma}^{t}|\mathbf{J}|\right) \mathrm{d} V_{0}=\int_{V_{0}} \frac{\mathrm{d} \mathbf{B}^{\mathrm{T}}}{\mathrm{d} q} \boldsymbol{\sigma}^{t}|\boldsymbol{J}| \mathrm{d} V_{0}+ \\
& \int_{V_{0}} \mathbf{B}^{\mathrm{T}} \frac{\mathrm{d} \boldsymbol{\sigma}^{t}}{\mathrm{~d} q}|\mathbf{J}| \mathrm{d} V_{0}+\int_{V_{0}} \mathbf{B}^{\mathrm{T}} \boldsymbol{\sigma}^{t} \frac{\mathrm{d}|\mathbf{J}|}{\mathrm{d} q} \mathrm{~d} V_{0}=0,
\end{aligned}
$$

where $V_{0}$ refers to the integration domain corresponding to one element. Most of the integral terms of the last expression are well-known and can be obtained by using the wellestablished strategies used for linear problems (see Wang et al. 1985). Nevertheless, the second integral term contains the expression $\mathrm{d} \sigma^{t} / \mathrm{d} q$ that includes the nonlinearity that characterizes the constitutive material model. All the difficulties of the sensitivity analysis for material nonlinear problems are concentrated in the computation of this term. Next we will see how, under some specific conditions, this term can be obtained through the use of the tangent constitutive matrix.

For a general nonlinear material model, during the equilibrium structural analysis the stresses at each point for a given pseudo-time $t$ can depend on the entire history of defor-

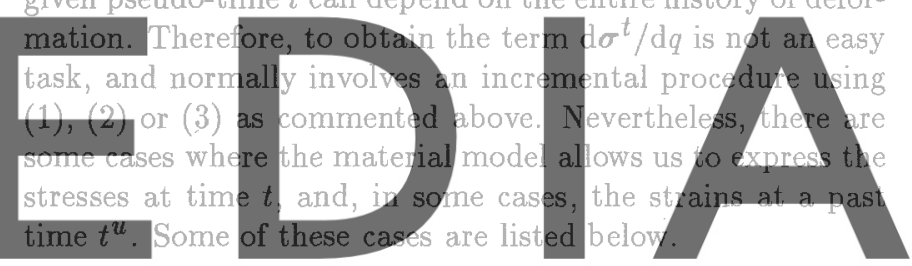

- Linear and nonlinear elastic models: this is an obvious dowałoad the version without the watermark

- Perfect plasticity models: this case was presented by Silva. et al. (1097).

- Damage models: this case will be shown in detail in the next section.

In a general plasticity case the stresses at each time $t$ can be expressed in terms of the total strains $\varepsilon^{t}$ at the same time $t$ and the plastic strains $\varepsilon_{p}^{t}$ at the same time as follows:

$\boldsymbol{\sigma}^{t}=\mathbf{D}\left(\varepsilon^{t}-\varepsilon_{p}^{t}\right)$

where $\mathbf{D}$ is the linear constitutive matrix. In order to obtain the plastic strain at time $t$ there are three possibilities.

1. If the stresses have not yet reached the yield surface the plastic strains are null and then (9) reduces to $\sigma^{t}=\mathbf{D} \varepsilon^{t}$.

2. When the stresses lie in the yield surface the plastic strain at time $t$ can be obtained in terms of the total strain $\varepsilon^{t}$ in a direct way by using the consistency equation and a radial return algorithm. In this case (9) holds, and $\varepsilon_{p}^{t}$ can be written in terms of $\varepsilon^{t}$.

3. When due to an unloading situation, the stresses go back to the interior of the space limited by the yield surface, the value of the plastic strain $\varepsilon_{p}^{t}$ at time $t$ is the same 
as the plastic strain $\varepsilon_{p}^{u}$ at the time where the unloading process started $t^{u}$. In this case (9) transforms into $\sigma^{t}=$ $\mathbf{D}\left(\varepsilon^{t}-\varepsilon_{p}^{u}\right)$.

As has just been shown, in the particular case of the perfect plasticity, the stresses at time $t$ can be expressed in terms of the strains at time $t$ and a set of internal variables $\kappa^{t}$ (plastic strains) which, in turn, depend on the strains at time $t$ and, in some situations, the strains at a past time $t^{u}$. This can be expressed as follows:

$\sigma^{t}=\sigma^{t}\left[\varepsilon^{t}, \kappa^{t}\left(\varepsilon^{u}, \ell_{c}\right)\right]$

In (10) a possible dependence with respect to a characteristic length $\ell_{c}$ has been considered. This is normally used if the material model contains a strain softening behaviour. The use of the characteristic length $\ell_{C}$ is necessary for the regularization of the solutions obtained from a smeared (continuous) model after the analysis of a cracking (discontinuous) phenomena, especially for cases with softening (see Oliver 1989)

On the other hand, in the case of a simple damage model $\kappa^{t}$ reduces to the damage parameter $d^{t}$ that characterizes the amount of existing damage, and then (10) can be written in the following way:

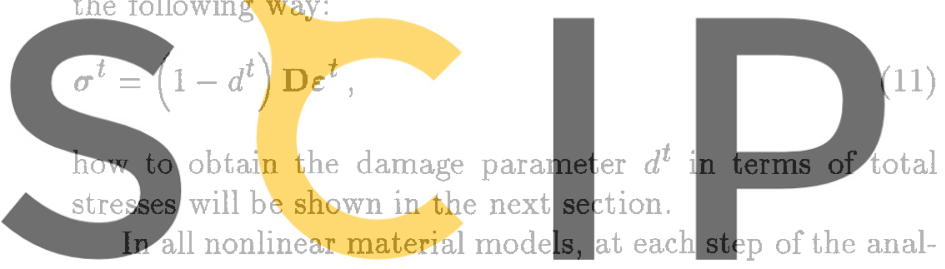
ysis the variation of the strains and stresses at the time $t$ are related through the tangent constitutive matrix. $D_{\text {T }}^{t}$ in the Registerffor free at https//wWw.scipedia.com to

$d \sigma^{t}=\mathrm{D}_{\mathrm{T}}^{t} d e^{t}$

Moreover, by taking (10) into account this matrix can be written as follows:

$\mathrm{D}_{\mathrm{T}}^{t}=\frac{\partial \boldsymbol{\sigma}^{t}}{\partial \boldsymbol{\varepsilon}^{t}}+\frac{\partial \boldsymbol{\sigma}^{t}}{\partial \boldsymbol{\kappa}^{t}} \frac{\mathrm{d} \boldsymbol{\kappa}^{t}}{\mathrm{~d} \varepsilon^{t}}$,

where all the derivatives must be evaluated at the time $t$. For instance, for the classical plasticity model of (9) the matrix is

$\mathbf{D}_{\mathrm{T}}^{t}=\mathbf{D}\left(1-\frac{\partial \varepsilon_{p}^{t}}{\partial \varepsilon^{t}}\right)$,

and for the damage model of (11) it is

$\mathrm{D}_{\mathrm{T}}^{t}=\mathrm{D}\left(1-d^{t}-\varepsilon \frac{{ }^{t} \partial d^{t}}{\partial \varepsilon^{\bar{t}}}\right)$.

If we now want to obtain the derivative of the stresses at time $t$ with respect to the design variable $q$, we can consider again (10) taking into account all the possible dependencies of the stresses with respect to the design variable $q$ in the following way:

$\boldsymbol{\sigma}^{t}=\boldsymbol{\sigma}^{t}\left\{\varepsilon^{t}(q), \kappa^{t}\left[\varepsilon^{t}(q), \varepsilon^{u}(q), \ell_{c}(q), q\right], q\right\}$.
Nevertheless, normally there is no explict dependence of either the trusses or the interal variables with respect to the design variable (this would be the case if, for instance, the Young's modulus or Poisson's ratio were a design variable). Therefore, (16) can be reduced to

$\sigma^{t}=\sigma^{t}\left\{\varepsilon^{t}(q), \kappa^{t}\left[\varepsilon^{t}(q), \varepsilon^{u}(q), \ell_{c}(q)\right]\right\}$.

Then we can differentiate the stresses with respect to the design variable as follows:

$\frac{\mathrm{d} \sigma^{t}}{\mathrm{~d} q}=\frac{\partial \boldsymbol{\sigma}^{t}}{\partial \varepsilon^{t}} \frac{\mathrm{d} \varepsilon^{t}}{\mathrm{~d} q}+$

$\frac{\partial \boldsymbol{\sigma}^{t}}{\partial \boldsymbol{\kappa}^{t}}\left(\frac{\partial \boldsymbol{\kappa}^{t}}{\partial \varepsilon^{t}} \frac{\mathrm{d} \boldsymbol{\varepsilon}^{t}}{\mathrm{~d} q}+\frac{\partial \boldsymbol{\kappa}^{t}}{\partial \varepsilon^{u}} \frac{\mathrm{d} \boldsymbol{\varepsilon}^{u}}{\mathrm{~d} q}+\frac{\partial \boldsymbol{\kappa}^{t}}{\partial \ell_{c}} \frac{\mathrm{d} \ell_{c}}{\mathrm{~d} q}\right)=$

$\left(\frac{\partial \sigma^{t}}{\partial \varepsilon^{t}}+\frac{\partial \sigma^{t}}{\partial \kappa^{t}} \frac{\partial \kappa^{t}}{\partial \varepsilon^{t}}\right) \frac{\mathrm{d} \varepsilon^{t}}{\mathrm{~d} q}+$

$\frac{\partial \sigma^{t}}{\partial \kappa^{t}}\left(\frac{\partial \kappa^{t}}{\partial \varepsilon^{u}} \frac{\mathrm{d} \varepsilon^{u}}{\mathrm{~d} q}+\frac{\partial \kappa^{t}}{\partial \ell_{c}} \frac{\mathrm{d} \ell_{c}}{\mathrm{~d} q}\right)$

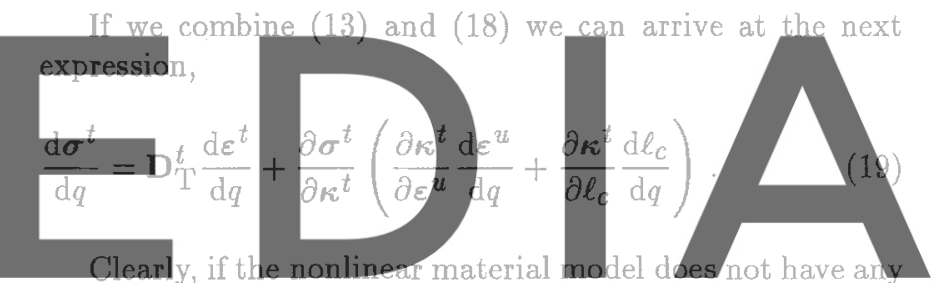

dependence with respect to the characteristic length, the last

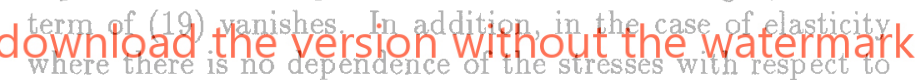

any internal variable, the terms where it appears are null Finally, the terms where $\varepsilon^{2 b}$ appears will be null, unless they correspond to an unloading situation. In this last case the tangent of the constitutive matrix $\mathbf{D}_{\mathrm{T}}^{t}$ will also correspond with the unloading situation.

On the other hand, we have to take into account that if a classical finite element discretized linear relation between the displacement field and the strains is assumed, we have

$\varepsilon^{t}=\mathbf{B u}$

where $\mathbf{u}^{t}$ is the nodal displacements vector at the time $t$. Differentiating (20) with respect to a design variable gives

$\frac{\mathrm{d} \varepsilon^{t}}{\mathrm{~d} q}=\frac{\mathrm{d} \mathbf{B}}{\mathrm{d} q} \mathbf{u}^{t}+\mathbf{B} \frac{\mathrm{d} \mathbf{u}^{t}}{\mathrm{~d} q}$

Now, if we substitute (19) and (21) into (8) and rearrange terms, we obtain the following expression:

$\int_{V_{0}} \frac{\mathrm{d}}{\mathrm{d} q}\left(\mathbf{B}^{\mathrm{T}} \boldsymbol{\sigma}^{t}|\mathbf{J}|\right) \mathrm{d} V_{0}=$

$\int_{V_{0}}\left(\frac{\mathrm{d} \mathbf{B}^{\mathrm{T}}}{\mathrm{d} q} \boldsymbol{\sigma}^{t}|\mathbf{J}|+\mathbf{B}^{\mathrm{T}} \boldsymbol{\sigma}^{t} \frac{\mathrm{d}|\mathbf{J}|}{\mathrm{d} q}\right) \mathrm{d} V_{0}+$ 


$$
\begin{aligned}
& \int_{V_{0}} \mathbf{B}^{\mathrm{T}}\left[\mathbf{D}_{\mathrm{T}}^{t}\left(\frac{\mathrm{d} \mathbf{B}}{\mathrm{d} q} \mathbf{u}^{t}+\mathbf{B} \frac{\mathrm{d} \mathbf{u}^{t}}{\mathrm{~d} q}\right)+\right. \\
& \left.\frac{\partial \boldsymbol{\sigma}^{t}}{\partial \boldsymbol{\kappa}^{t}}\left(\frac{\partial \boldsymbol{\kappa}^{t}}{\partial \varepsilon^{t}} \frac{\mathrm{d} \varepsilon^{u}}{\mathrm{~d} q}+\frac{\partial \boldsymbol{\kappa}^{t}}{\partial \ell_{c}} \frac{\mathrm{d} \ell_{c}}{\mathrm{~d} q}\right)\right]|\mathbf{J}| \mathrm{d} V_{0},
\end{aligned}
$$

Now, it we substitute (22) into (7) we obtain the following matrix expression:

$\mathbf{K}_{\mathrm{T}}^{t} \frac{\mathrm{d} \mathbf{u}^{t}}{\mathrm{~d} q}=\mathbf{f}^{t *}$

where $\mathbf{K}_{\mathrm{T}}^{t}$ is the tangent stiffness matrix and $\mathbf{f}^{t *}$ is a pseudo load vector, both evaluated at the time $t$. Their expressions are
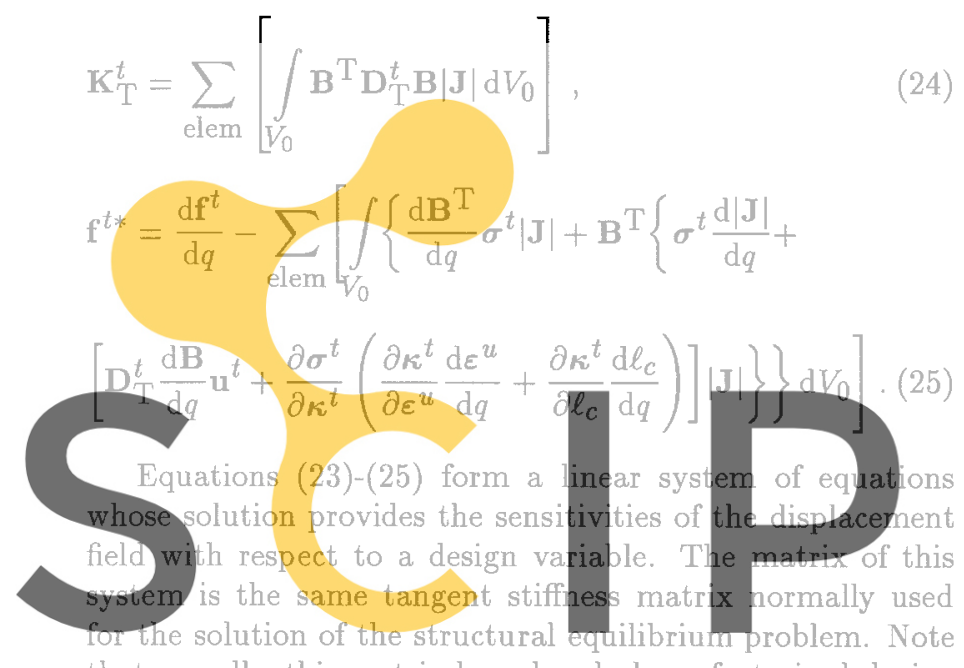
that, usually, this matrix has already been factorized during

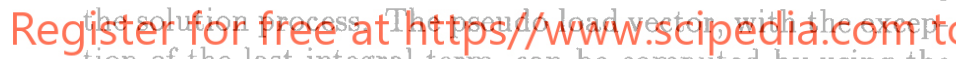
tion of the last integral term, can be computed by using the same techniques as in the case of linear structural problems. This last integral term can be easily computed by using the tangent constitutive matrix.

It must be emphasized that the displacements and the tangent stiffness matrix that appear in (23)-(25) are obtained during the solution of the structural equilibrium problem in the usual incremental way. This means that they incorporate all the dependencies with respect to the strains and stresses that the nonlinear material model assigns to them.

In consequence, despite the fact that traditionally the structural sensitivity analysis for nonlinear problems is obtained in an incremental way, last expressions show that, in the mentioned particular cases, this analysis can be obtained in a direct way after the solution of the structural equilibrium problem is obtained.

\section{Damage material model}

Continuous damage material models are based on the use of an internal variable $d^{t}$ that controls the mechanical behaviour of the material. The constitutive equation of the simplest damage model depends on a single parameter $d^{t}$ in the way expressed in (11),

$$
\sigma^{t}=\left(1-d^{t}\right) \mathbf{D} \varepsilon^{t}=\left(1-d^{t}\right) \mathbf{D B u}{ }^{t}, \quad 0 \leq d^{t} \leq 1 \text {. }
$$

The damage parameter evolves in terms of the strain state. In this work the following evolution equation has been used by Oliver et al. (1990):

$d^{t}=1-\frac{\tau^{*}}{v^{t}} \mathbf{e}^{A\left(1-\frac{v^{t}}{\tau^{*}}\right)}, \quad v^{t}=\max _{s \in[0, t]}\left(\tau^{*}, \tau^{s}\right)$

where $\tau^{t}$ is a norm of the stress state given by

$\tau^{t}=[1+r(n-1)] \sqrt{\left(\sigma_{1}^{e}\right)^{2}+\left(\sigma_{2}^{e}\right)^{2}+\left(\sigma_{3}^{e}\right)^{2}}$

with

$r=\sum_{i=1}^{3} \frac{\left\langle\sigma_{1}^{e}\right\rangle}{\left|\sigma_{i}^{e}\right|},\left\langle\sigma_{i}^{e}\right\rangle=\frac{1}{2}\left[\sigma_{i}^{e}+\left|\sigma_{i}^{e}\right|\right], n=\frac{f_{c}}{f_{t}}$,

where $\sigma^{e}=\mathbb{D} \varepsilon$ are the elastic stresses, $\sigma_{i}^{e}$ are the principal elastic stresses and $n$ is the ratio between the maximum allowable stresses for compression $\left(f_{c}\right)$ and traction $\left(f_{t}\right)$. Finally, $\tau^{*}$ is the threshold value of this norm (a material property) above which the material starts to damage and $A$ is a softening parameter which depends on the characteristic length of the elements $\ell_{c}$ (a measure of the mesh element size), fracture energy $G_{f}$, maximum traction stress $f_{t}$ and Young's modulus

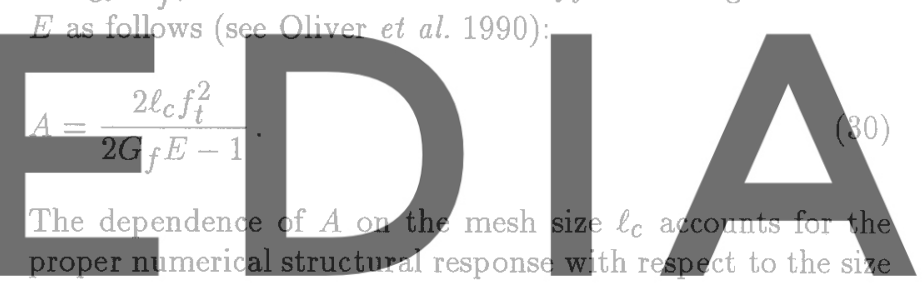
of the finite elements. As mentioned earlier, this dependence is necessary when a condinuous. displacement field (smeared

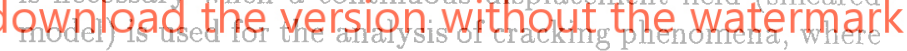
the displacements are known to become discontinuous (see Oliver 1989). This means that, in practice, the constitutive equation at each element depends on its size. In the case of shape sensitivity analysis this effect must be taken into account because a change on the structural shape can affect the element sizes and, in consequence, also the stress values.

Expression (27) indicates the following.

- The behaviour of the material is initially elastic $\left(d^{t}=1\right)$ until the norm of the stress rate reaches the threshold value $\tau^{*}$.

- Once the norm of the stress state become higher than the threshold value damage parameter $d^{t}$, an increase of the applied load results in an increase of the norm of the stress state. Therefore, in this situation the value of $v^{t}$ corresponds with the value of the norm of the stress state $\tau^{t}$, and the value of the damage parameter at time $t$ can be obtained in terms of the strains at time $t$.

- In the case of an unloading situation of a damaged material, the value of $v^{t}$ corresponds the the value of the norm of the stress state $\left(v^{t}=\tau^{u}\right)$, obtained at the previous time $t^{u}$ when the unloading process started. This norm is obtained in terms of the stresses at that time $\varepsilon^{u}$. In this case, the value of the damage parameter at time $t$ can be obtained in terms of the strains at time $t^{u}$. 
To the authors' knowledge, the above comments are valid for all the damage material models. As can be seen from these comments, (19) is valid for this damage model because the stresses at time $t$ can be obtained in terms of the strains at time $t$, the strains at time $t^{u}$, and a characteristic length. Therefore, the structural sensitivity analysis for the material model can be obtained using (23) to (25).

If we compute the value of the different derivatives that appear in the last term of (25), we have

$$
\begin{aligned}
& \frac{\partial \boldsymbol{\sigma}^{t}}{\partial \boldsymbol{\kappa}^{t}}=\frac{\partial \boldsymbol{\sigma}^{t}}{\partial d^{t}}=-\mathbf{D} \varepsilon^{t}=-\mathbf{D B} \mathbf{u}^{t}, \\
& \frac{\partial \boldsymbol{\kappa}^{t}}{\partial \varepsilon^{u}}=\frac{\partial d^{t}}{\partial \varepsilon^{u}}=\frac{\partial d^{t}}{\partial \tau^{u}} \frac{\partial \tau^{u}}{\partial \varepsilon^{u}} .
\end{aligned}
$$

The derivatives of the last expression can be obtained through the direct derivation of (26) and (27). They need only be taken into account in unloading situations and already damaged material. If this is the case, the term $\mathrm{d} \varepsilon^{u} / \mathrm{d} q$ must be computed at the time $t^{u}$ when the unloading process starts. This term can be obtained from the sensitivity of the displacements at time $t^{u}$ using

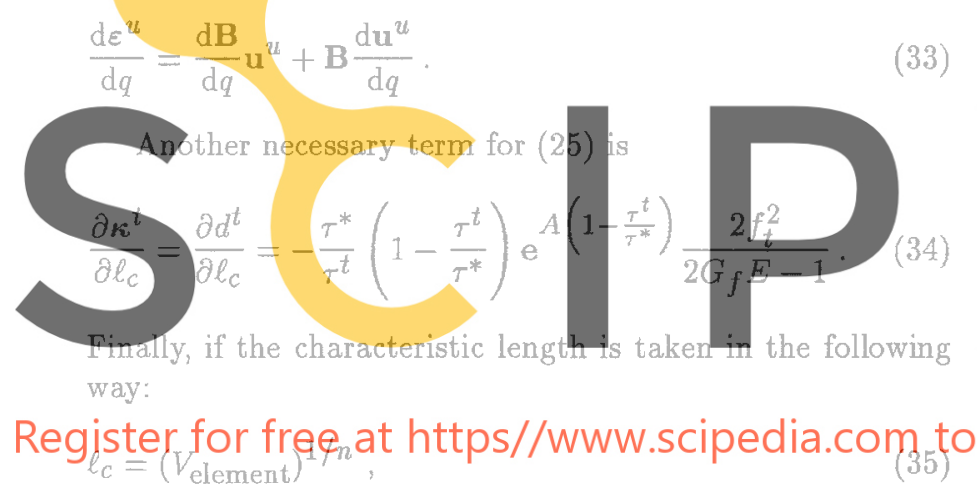

where $n$ takes the values 1,2 or 3 for one, two or threedimensional problems, respectively, then we have,

$\frac{\mathrm{d} \ell_{c}}{\mathrm{~d} q}=\frac{1}{n \ell_{c}^{n-1}} \frac{\mathrm{d} V_{\text {element }}}{\mathrm{d} q}$.

Finally, if we substitute (31) to (36) into (25) we obtain the final expression for the pseudo load vector corresponding to this type of material model. The final expression is not written here due to its excessive length.

\section{Discussion and strategy for sensitivity analysis with strain-softening problems}

The solution of (23)-(25), as well as any other method based on a traditional incremental approach, provides the sensitivities of the structural behaviour assuming that when the structural shape is perturbed the loads remain constant. These sensitivities are the derivatives of the structural behaviour with respect to the design variables, and they are obtained assuming an infinitesimal perturbation of these design variables. Nevertheless, the sensitivity analysis is often used for the first-order prediction of the nonlinear behaviour of a modified structure that has been obtained by the application of a finite perturbation. By using this approach, one can estimate the answer of the new structure under the same load level as the original structure. This means that the behaviour of a structure that we would obtain after a finite perturbation of the design variable $q$ can be approximated by the following expression:

$\mathbf{u}(q+\Delta q) \approx \mathbf{u}(q)+\frac{\mathrm{d} \mathbf{u}}{\mathrm{d} q} \Delta q$

Figure 1 shows the meaning of this type of projection assuming that we have the full load displacement curve for the original and the perturbed structures. In this figure the continuous line shows the equilibrium response curve of a given structure under a specific loading history, and the dashed line shows the estimation of the corresponding equilibrium response curve for a perturbed structure under the same loading history.

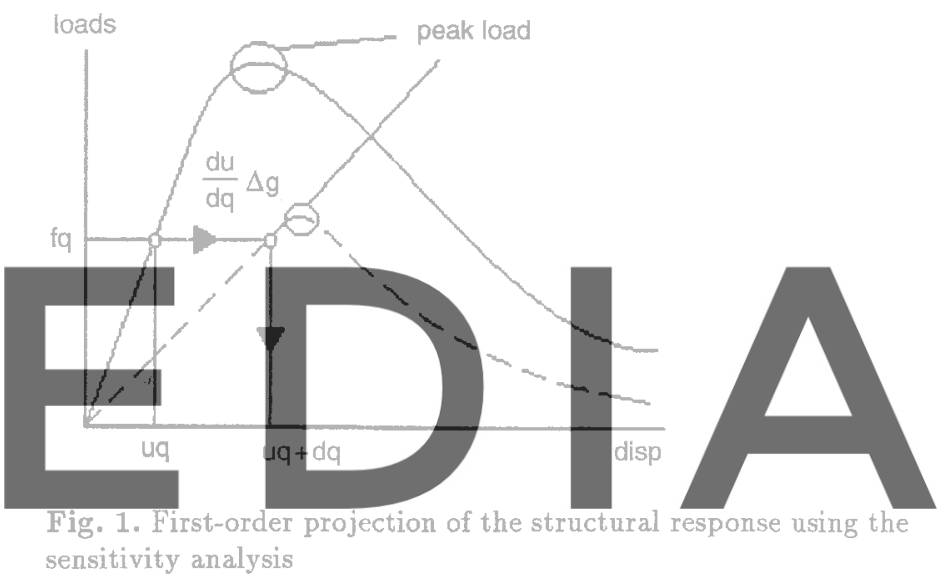

download the version without the watermark

When the structural behaviour includes strain softening, the use of this type of projection for finite perturbations can become meaningless. In principle we do not know if a certain finite modification of a design variable will increase or decrease the peak load of the structural response. In particular, if the peak load decreases, it makes no sense to use a standard sensitivity analysis to project the structural behaviour to the perturbed one. A clear example of this situation is shown in Fig. 2. Note that in this case a projection form the highest loaded equilibrium points of the original structure drive to situations where the perturbed structure cannot be equilibrated.

On the other hand, in cases where the perturbed structure has a higher peak load than the original one, it would not be possible to predict its value. This case is shown in Fig. 3, where we cannot estimate any of the highest loaded equilibrium points of the perturbed structure by a horizontal projection from the original one.

It seems clear that in the two mentioned pathological situations, the projections from the original structure to the perturbed one should involve a variation not only of the displacement field but also of the load level. This type of projection is named desirable projection in Figs. 2 and 3 . In this case it is not enough to know the sensitivities of the unknown variables of the equilibrium equations. It is also necessary to know the sensitivities of the load forces. 


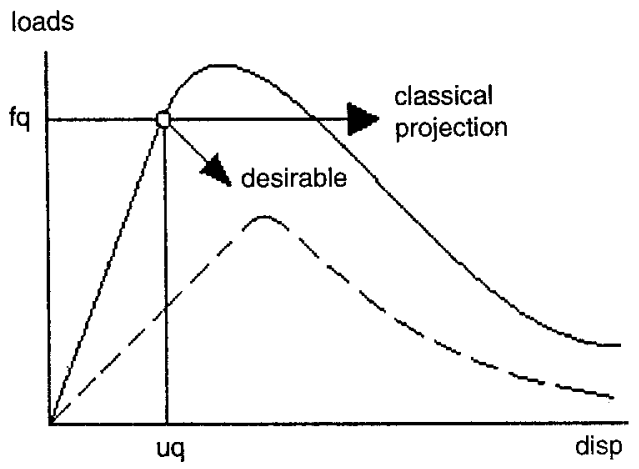

Fig. 2. Nonfeasible projections of the structural response by using a constant load sensitivity analysis

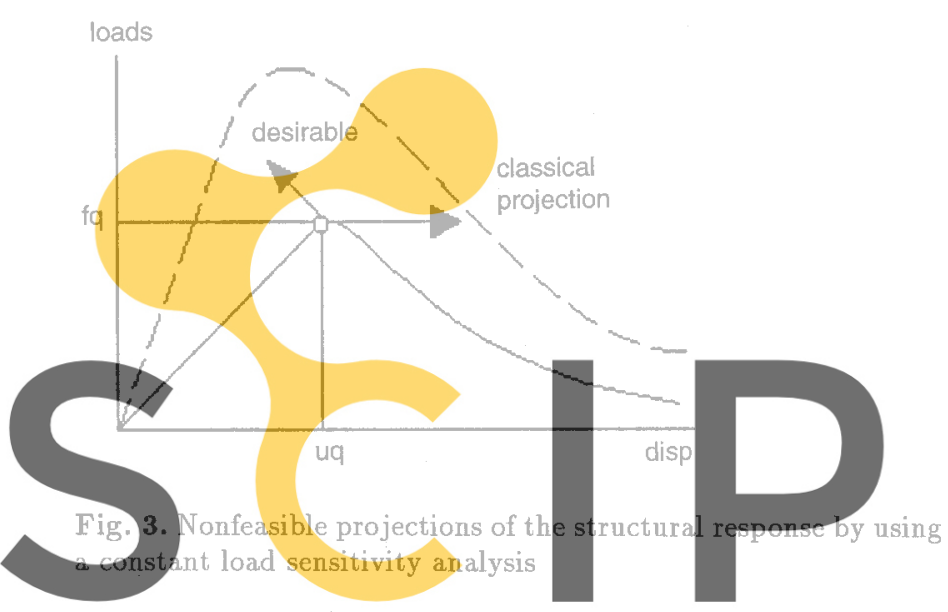

It should be emphasized that this type of problem ap-

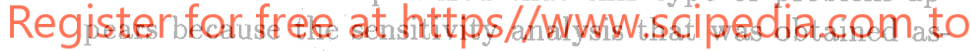
suming an infinitesimal perturbation of a design variable is used to predict the behaviour of a new structure that is defined through a finite perturbation of a design variable. In fact, when infinitesimal perturbations are applied there are no finite variations of the peak load.

From the mechanical point of view, structural problems with strain softening present a reduction of the structural resistance after the peak load equilibrium point. After this situation it is not possible to increase the magnitude of the loads applied to the structure. In consequence, a classical analysis strategy based on an incremental application of the loads does not allow studying the structural behaviour after the peak load point.

On the other hand, the displacement field can always be increased producing new equilibrium states in the structural response curve, even if the structural behaviour contains strain softening. This allows the use of arc-length methods for the study of the structural behaviour of strain-softening problems after the peak load. This type of method is based on the simultaneous accomplishment of the equilibrium equations and some conditions about the displacement field (Crisfield 1991). Both conditions assure new equilibrium points in the displacement load curve.

Taking into account that the solution strategies of this type of problem are based on the use of a fixed arc-length for each equilibrium point instead of a fixed load level, it seems logical to use this type of condition to predict the behaviour of a perturbed structure. In this case, the projections will be made assuming that the arc-length condition, and not the load level, will remain constant. This approach has the advantage that no assumption is made on the peak load of the perturbed structure; in addition, it is completely consistent with the equilibrium equations.

Arc-length methods are based on the simultaneous accomplishment of the following two equations:

$\sum_{\text {elem }} \int_{V} \mathbf{B}^{t} \boldsymbol{\sigma} \mathrm{d} V-\lambda \mathbf{f}=0$

$g(\mathbf{u}, \lambda)=0$

where $\lambda$ is a load factor parameter that controls the magnitude of the load applied at each equilibrium point and (39) is a condition on the values of the displacement field at the same point. Traditionally, this condition is applied on the value of the displacement of a single node or on the value of a norm of the nodal displacement vector. Compared with a classical incremental approach the arc-length methods introduce the additional unknown $\lambda$ and the additional equation (39)

The sensitivity analysis of the arc-length equations in-

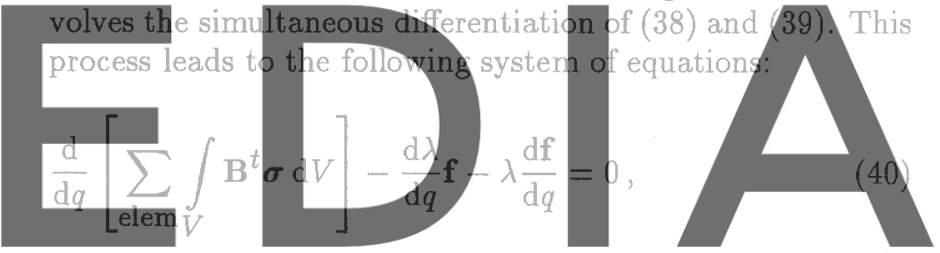

$\underline{\mathrm{d} g}=\frac{\partial g}{\partial \theta}+\underline{\partial g} \underline{\mathrm{d} u}+\frac{\partial g}{\partial \mathrm{d} \lambda}$

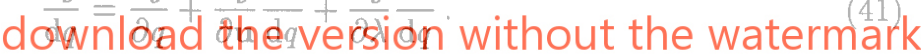

Equation (40) is similar to (6) with the addition of some terms to the pseudo-load vector. On the other hand, the different terms of (41) are very easily obtained by differentiation of the arc-length condition.

The simultaneous solution of (48) and (49) can be grouped in the following, more compact, matrix form:

$\left[\begin{array}{cc}\mathbf{K}_{\mathrm{T}} & -\mathbf{f} \\ \partial g / \partial \mathbf{u} & \partial g / \partial \lambda\end{array}\right]\left\{\begin{array}{l}\mathrm{d} \mathbf{u} / \mathrm{d} q \\ \mathrm{~d} \lambda / \mathrm{d} q\end{array}\right\}=\left\{\begin{array}{c}\mathbf{f}^{*} \\ 0\end{array}\right\}$.

The solution of (42) provides the sensitivities of the displacements and the load factor parameter. If these sensitivities are used to project the structural behaviour by the perturbation of a design variable, it will produce a first-order estimation of the displacements and the load level of the new structure assuming the accomplishment of the same arc-length condition as the original one. This estimation will be obtained by using the following expressions:

$$
\begin{aligned}
& \mathbf{u}(q+\Delta q) \approx \mathbf{u}(q)+\frac{\mathrm{d} \mathbf{u}}{\mathrm{d} q} \Delta q, \\
& \lambda(q+\Delta q) \approx \lambda(q)+\frac{\mathrm{d} \lambda}{\mathrm{d} q} \Delta q .
\end{aligned}
$$

On the other hand, it must be mentioned that the addition of the arc-length condition to the equilibrium equation 
leads to the system of equations (42) that is no longer symmetric. Fortunately, due to the fact that only the last row and the last column are nonsymmetric it is possible to use cheap iterative strategies that take full advantage of the factorization of the original tangent stiffness matrix, see Gil (1996) for more details.
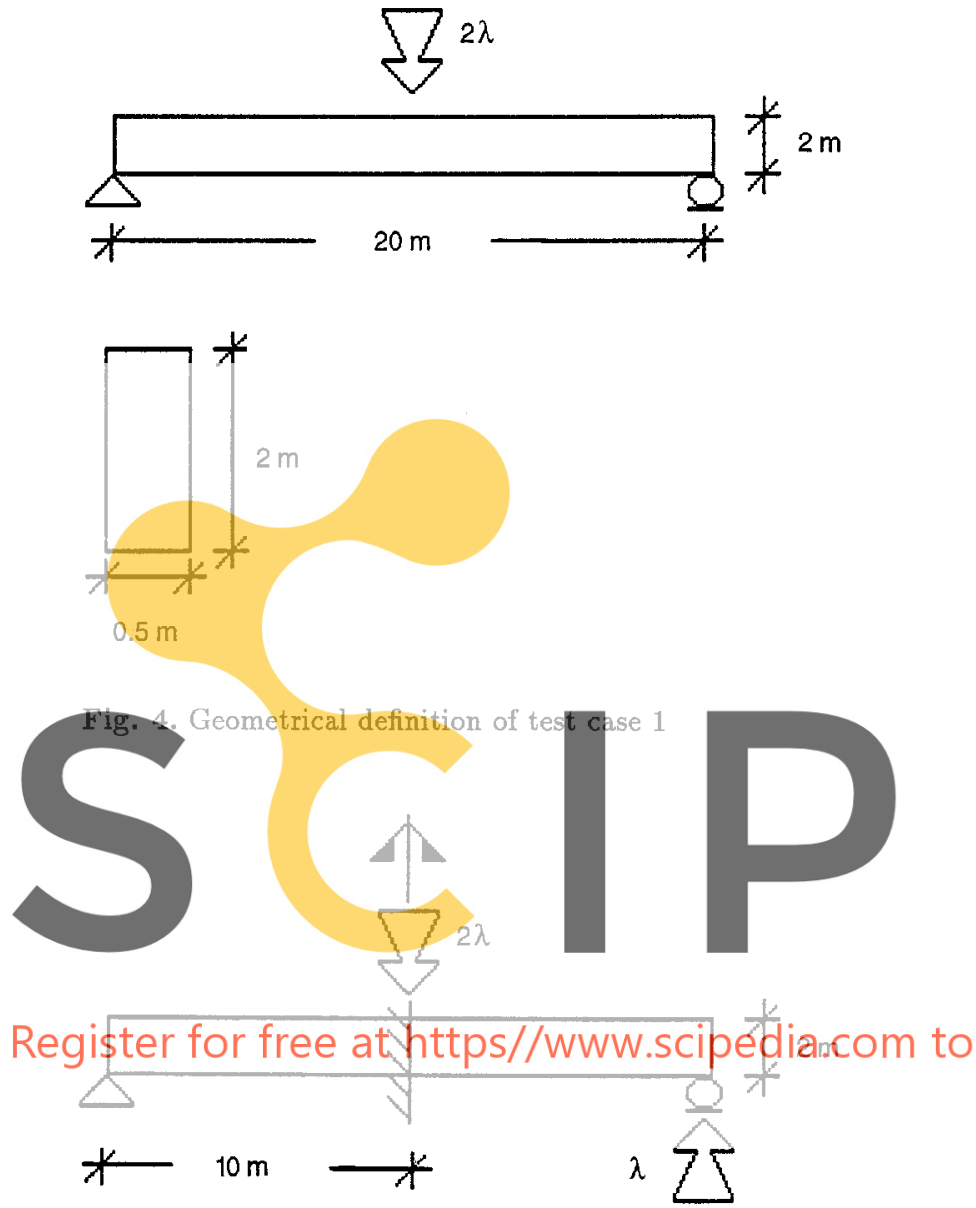

Fig. 5. Geometrical definition of test case 1

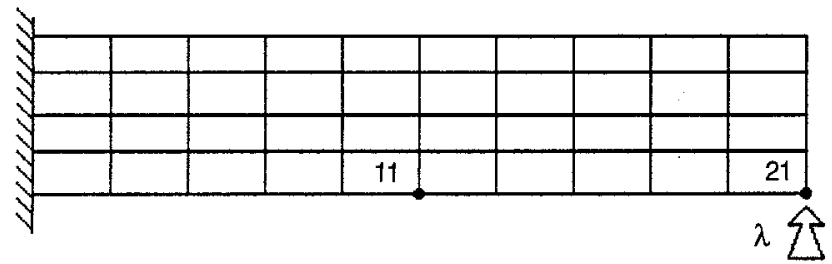

Fig. 6. Finite element mesh for test case 1

In addition, it should be mentioned that the strategy presented could be applied to any type of nonlinear structural problem, even if it has no strain-softening effect.

It should be noted that this procedure provides the design sensitivity analysis of the structural response, and this allows us to predict the complete structural response path (or, eventually, part of it), which can contain the new peak load. Nevertheless, it does not provide the design sensitivity

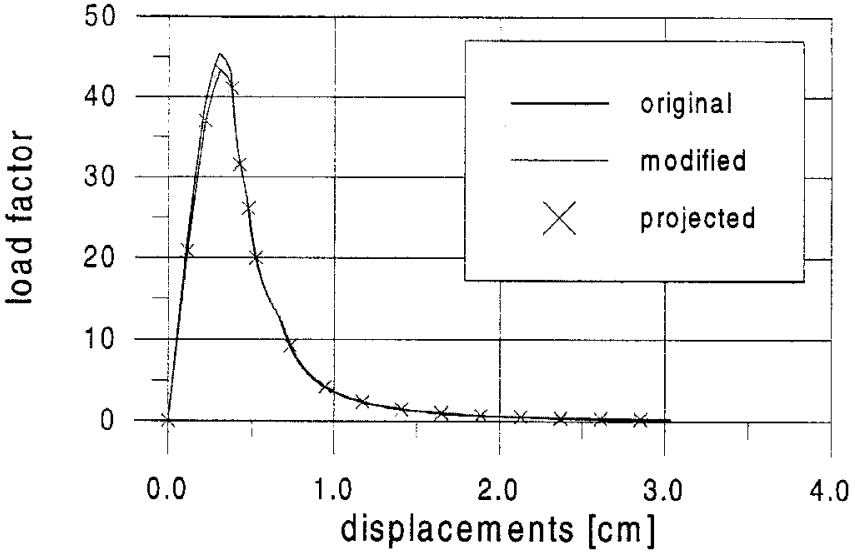

Fig. 7. Superposition of the displacement load curves corresponding to the original, the modified and the projected structures for test case 1

analysis of the peak load. How to obtain this last sensitivity is a different problem and is not treated here.

To the authors' knowledge, a similar approach was first proposed by Kleiber and Hien (1996) and it was applied to the sensitivity analysis of geometrically nonlinear problems. In the present work, this technique has been applied to struc

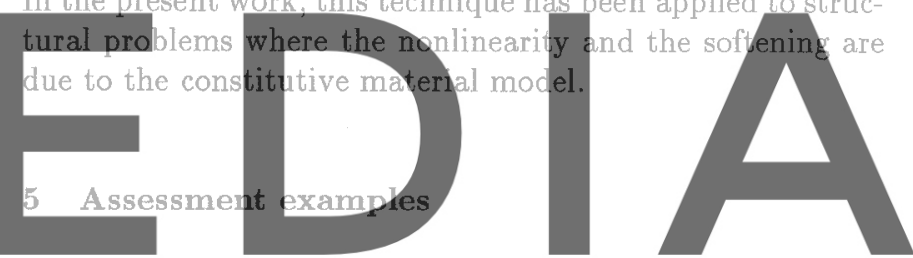

The quality and the reliability of the formulations proposed in this paper are assessed here through the resolution of two

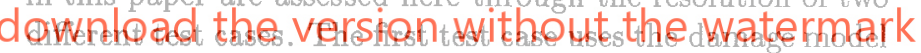
with strain softening for the analysis of a two-dimensional beam under a bending moment. The second test case uses the same material model for the analysis of a short beam with a variable cross-section.

\subsection{Test case 1. Two-dimensional beam under a bending moment}

This test case studies the quality of the proposed formulation for the case of a concrete iso-static beam with bending behaviour. The selected design variable is the thickness of the beam. This test case shows the application of the presented formulation to predict the behaviour of a beam that, due to building errors, has a thickness smaller than the designed and analysed one. This test case is analysed with a damage model with strain softening and the main aim is to predict the modification of the peak load produced by the building error.

The geometry and the applied load for this test case are described in Figs. 4 and 5 . Figure 5 also describes the symmetry approach used for the analysis of the structural problem.

The various data used for the structural analysis is given below.

- A plane stress model with a depth of $50 \mathrm{~cm}$ has been assumed. 


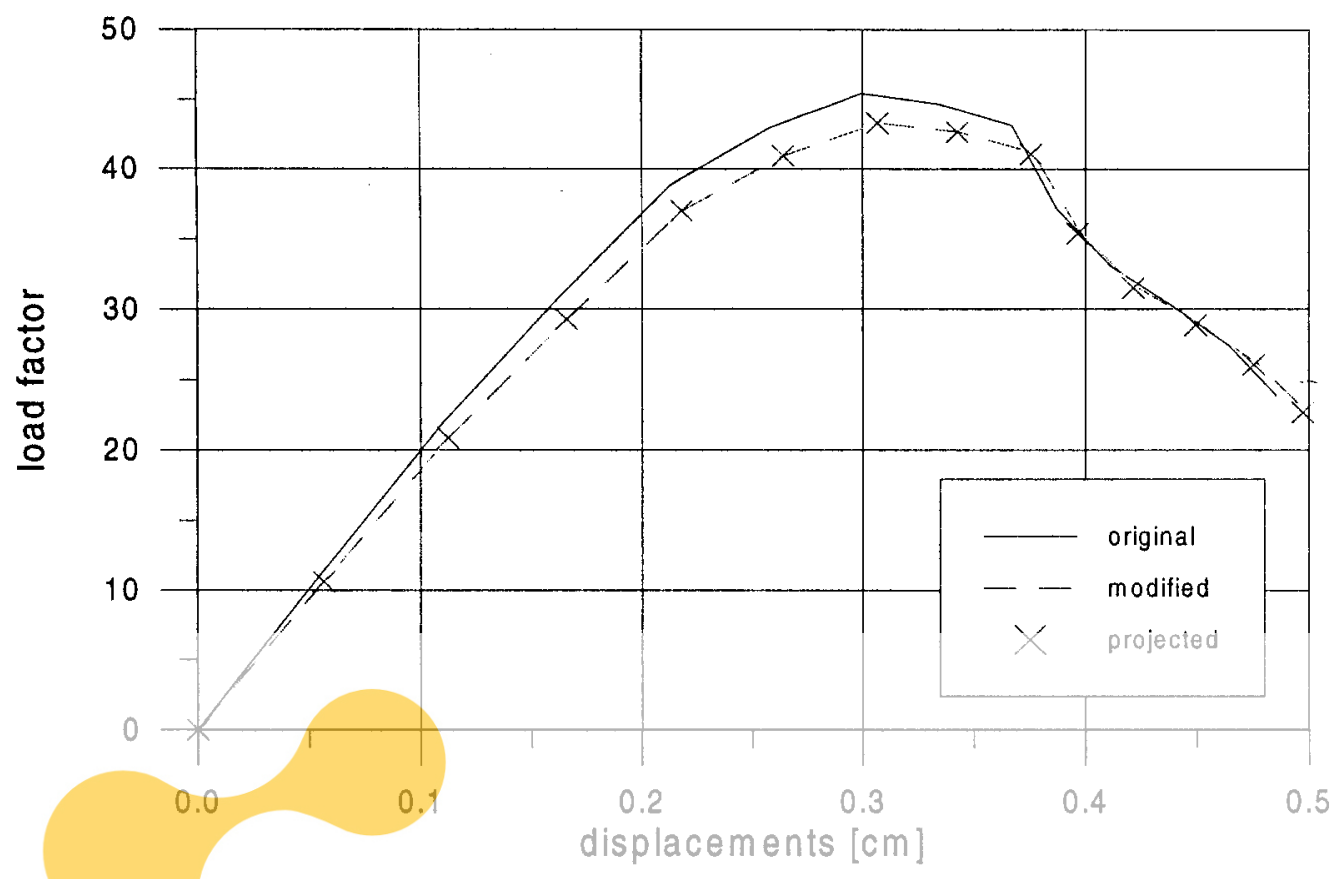

Fig. 8. Detail of the zone with maximum applied load

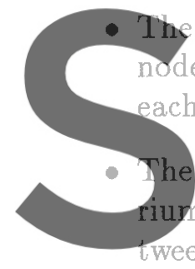

arc-length method controli 11 (see Fig. 6) with increm ach step of the solution proc

The convergence criteria for problem have been defined solution of the equilibf the ratio benorm of the external forces. This ratio has been limited to $1 \%$. The

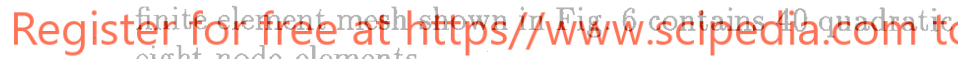
eight-node elements.

- The initial design variable is the thickness of the beam.

- The material properties are as shown in Table 1.

Table 1. Material properties for test case 1

\begin{tabular}{lll}
\hline Young's modulus $E$ & $2.110^{6}$ & $\mathrm{KN} / \mathrm{m}^{2}$ \\
\hline Poisson's ratio & 0.2 & \\
\hline Maximum compression stress & $2.010^{2}$ & $\mathrm{KN} / \mathrm{m}^{2}$ \\
\hline Maximum traction stress & 500.0 & $\mathrm{KN} / \mathrm{m}^{2}$ \\
\hline Fracture energy & 200.0 & $\mathrm{~J} / \mathrm{m}^{2}$ \\
\hline
\end{tabular}

Two different analyses have been performed. The first one corresponds to the original structure. For this structure the sensitivities of the displacements with respect to the design variable have been computed at each equilibrium point. The second analysis corresponds to a modified (perturbed) structure that has been obtained by applying a reduction of

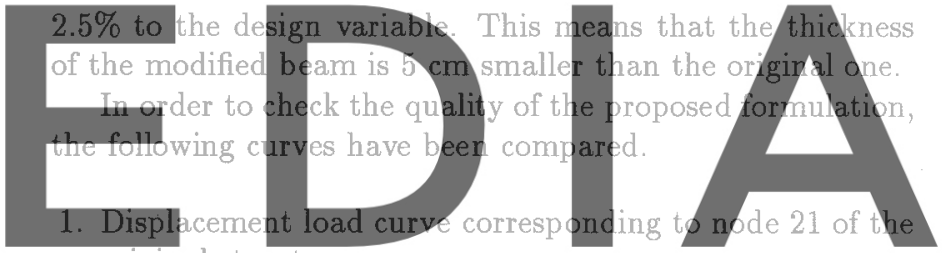

original structure.

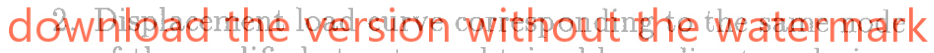
of the modified structure obtained by a direct analysis.

3. Displacement load curve corresponding to the same node of the modified structure obtained by a first-order projection [see (43)-(44)] using the results of the original one and its sensitivities.

The comparison of the response curves between the original, modified and projected structural behaviour is shown in Fig. 7. It should be noted that the curves projected and modified superpose quite well. This reveals a good behaviour of the proposed formulation for this test case. In particular, the projected curve allows a very good engineering estimation of the ultimate load of the modified structure (see Fig. $8)$.

\subsection{Test case 2. Two-dimensional short cantilever beam}

In this test case, the structural problem consists of a small cantilever beam with a variable cross-section (see Fig. 9).

The various data used for the structural analysis is shown in Table 2.

The various data used for the structural analysis is as follows.

- A damage constitutive material model as described in Section 3 . 
- Plane stress model equations assuming a thickness of 1 $\mathrm{cm}$.

- An Arc-length controlling the displacement of node 34 (see Fig. 10) allowing for an increment in its displacement norm of $\Delta 1=3.510^{-4} \mathrm{~cm}$ at each step of the solution process.

- The same convergence criteria as in the previous test cases.

- The design variable is the cross-section of the structure.

- A mesh of 12 eight-node quadratic elements has been chosen.

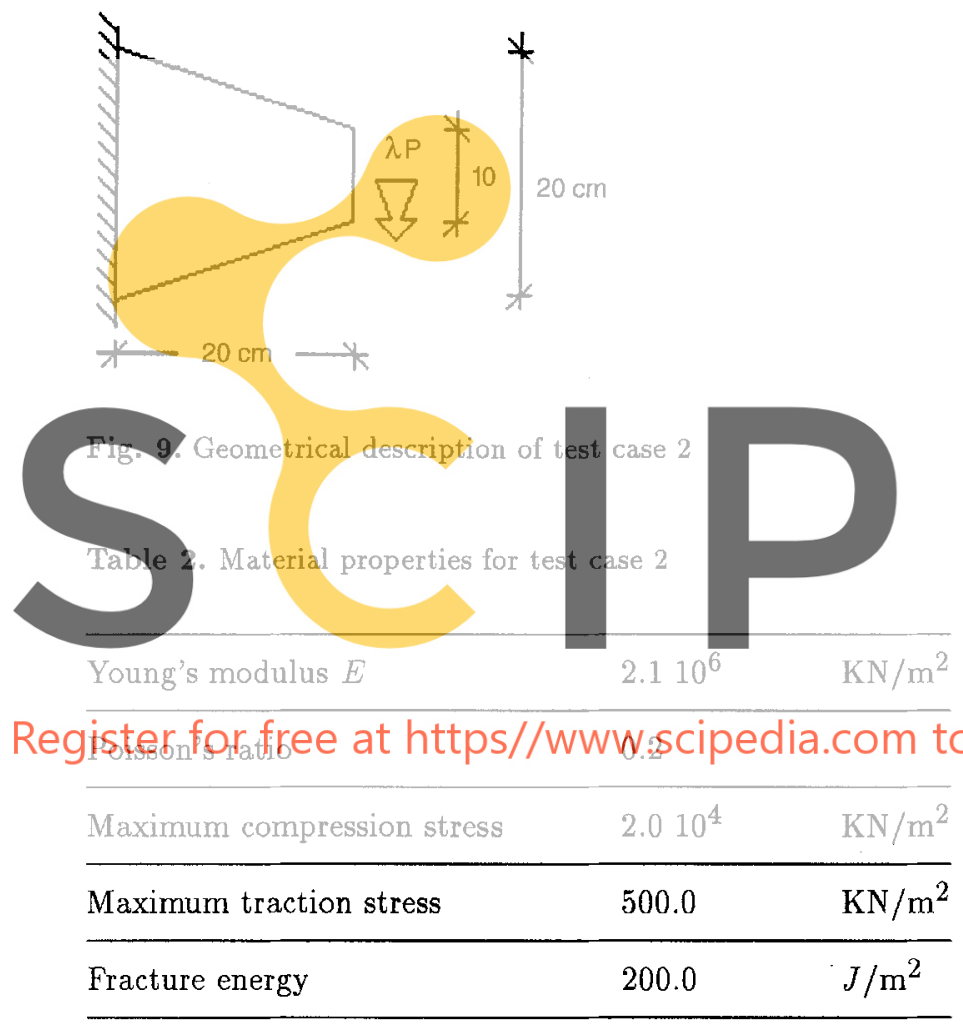

Similarly as in test case 1 two different analyses have been performed. The first analysis and sensibilities correspond to the original structure. The second one corresponds to a modified (perturbed) structure that has been obtained by applying a uniform increment of the cross-section. This means that the modified structure is $6.6 \%$ larger in surface than the original one.

With the same types of curves as in the previous test cases, Fig. 11 shows that the projected curve reproduces very well the behaviour of the perturbed structure. In fact, the coincidence of curves projected and modified is almost perfect.

\section{Conclusions}

Differentiation of the discretized global equilibrium equation allows a very satisfactory evaluation of the sensitivities of the

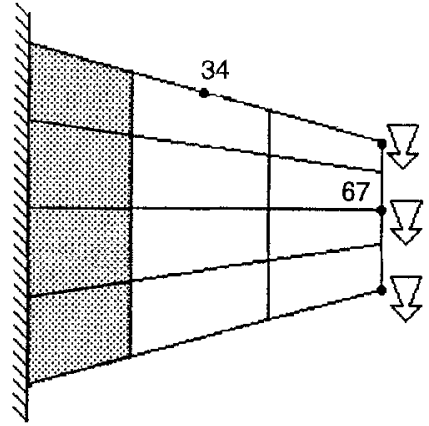

Fig. 10. Mesh and structural model, the coloured elements have a lower yield stress in order to localize the damage

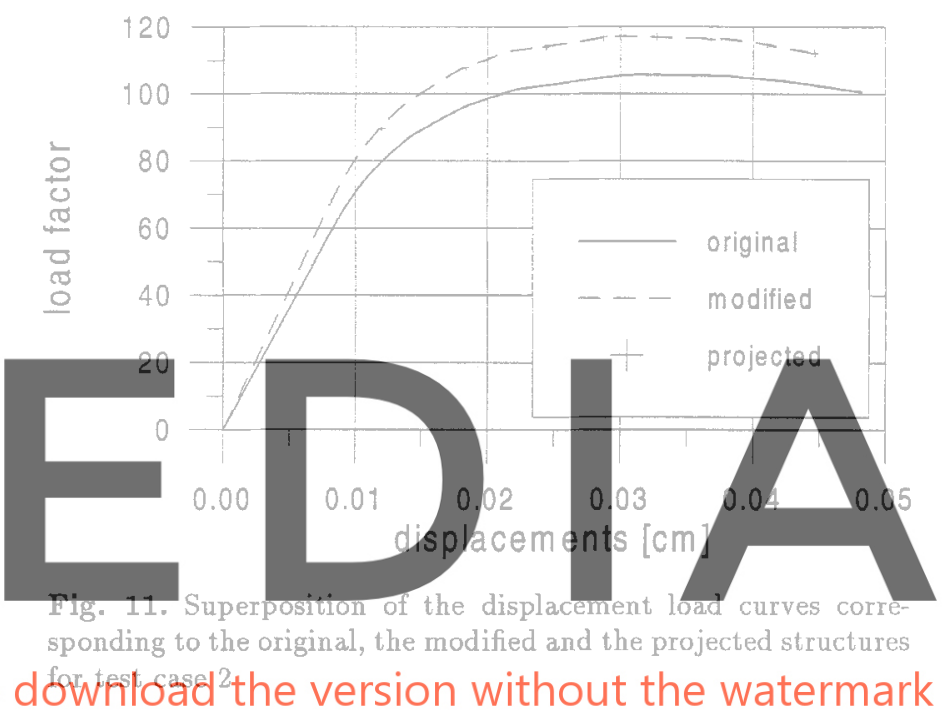

structural behaviour when a danage material model is used. This approach is based on the use of the tangent constitutive matrix and does not make use of any incremental approach.

The simultaneous differentiation of the equilibrium equations and the arc-length condition leads to a new strategy for the evaluation of the structural sensitivities that solves the projection problems when the structural behaviour presents strain-softening.

The quality of the formulation proposed here for the sensitivity analysis of structures containing damage models, together with the inclusion of the arc-length condition has been assessed through the resolution of different test cases. The results shown in all test cases are very satisfactory.

In particular, some examples show the good possibilities of the proposed sensitivity analysis for the study of situations where, due to a pathological situation, the finally built structure is not coincident to the orignally designed one.

\section{Acknowledgement}

The authors would like to express their gratitude to Prof. $\mathrm{X}$ avier Oliver for his very interesting suggestions and support during the development of the present work. 


\section{References}

Arora, J.S.; Cardoso, J.B. 1992: Variational principle for shape design sensitivity analysis. AIAA J. 30, 538-547

Cardoso, J.B.; Arora, J.S. 1988: Variational method for design sensitivity analysis in nonlinear structural mechanics $A I A A J \mathbf{2 6}$ 595-603

Choi, K.K.; Santos, J.L.T. 1987: Design sensitivity analysis of non-linear structural systems. Part 1 : Theory. Int. J. Num. Meth. Engrg. 24, 2039-2055

Crisfield, M.A. 1991: Non-linear finite element analysis of solids and structures. John Wiley \& Sons

Gil, L. 1996: Análisis de sensibilidad con comportamiento no lineal del material y su aplicación en el diseño asistido de estructuras. Ph.D. Thesis, ETS Ingenieros de Caminos, Canales y Puertos. Universitat Politècnica de Catalunya, Barcelona

Haslinger, J,; Mäkinen, R. 1992: Shape optimization of elastoplastic bodies under plane strains: sensitivity analysis and numerical implementation. Struct. Optim. 4, 133-141

Kleiber, M. 1993: Shape and non-shape structural sensitivity analysis for problems with any material and kinematic non-linearity. Comp. Meth. Appl. Mech. Ef Engrg. 108, 73-97

Kleiber, M.; Hien, T.D.; Postek, E. 1994: Incremental sensitivity

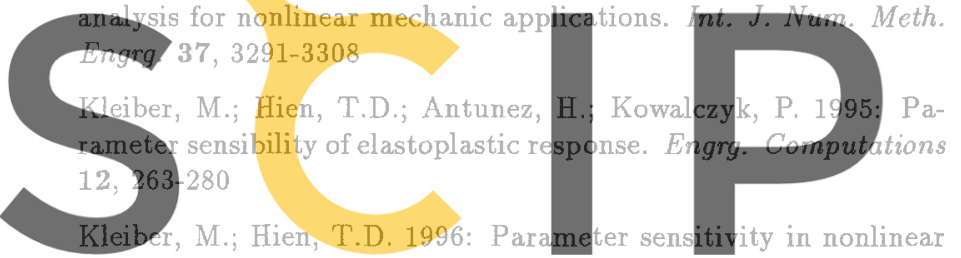
dynamics, structural stability and plasticity. In: Doblaré, M. Correas, J.M.; Alarçon, E.; Gavete; L.; Pastor, M. (eds.) Proc.

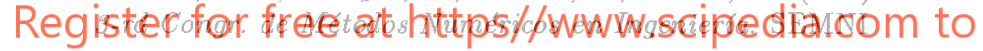

Oliver, J. 1989: A consistent characteristic length for smeared cracking models. Int. J. Num. Engrg. 28, 461-474

Oliver, J.; Cervera, M.; Oller, S.; Lubliner, J. 1990: A simple damage model for concrete, including long term effects. Proc. 2-nd Int. Conf. on Computer Aided Analysis and Design of Concrete Structures (held in Zell Am See, Austria), Vol. 2, pp. 945-958

Ryu, Y.S., Haririan, M.; Wu, C.C.; Arora, J.S. 1985: Structural design sensitivity analysis of nonlinear response. Comp. \& Struct. 21, 245-255

Santos, J.L.T.; Choi, K.K. 1992: Shape design sensitivity analysis of non-linear structural systems. Struct. Optim. 4, 23-35

Silva, C.E.K.; Hinton, E.; Vaz, L.E.; Sienz, J. 1997: 2D shape optimization with rate-independent elastoplasticity. In: Owen, D.R.J.; Oñate, E.; Hinton, E. (eds.) Proc. Computational Plasticity, Fundamentals and Applications, pp. 836-852. CIMNE

Tsay, J.J.; Arora, J.S. 1990: Nonlinear structural design sensitivity analysis for path dependent problems. Part 1: General theory. Comp. Meth. Appl. Mech. Engrg. 81, 183-208

Tsay, J.J.; Cardoso, J.E.B.; Arora, J.S. 1990: Nonlinear structural design sensitivity analysis for path dependent problems. Part 2: Analytical examples. Comp. Meth. Appl. Mech. Engrg. 81, 209-228

Vidal, C.A.; Haber, R.B. 1993: Design sensitivity analysis for rateindependent elastoplasticity. Comp. Meth. Appl. Mech. Engrg.

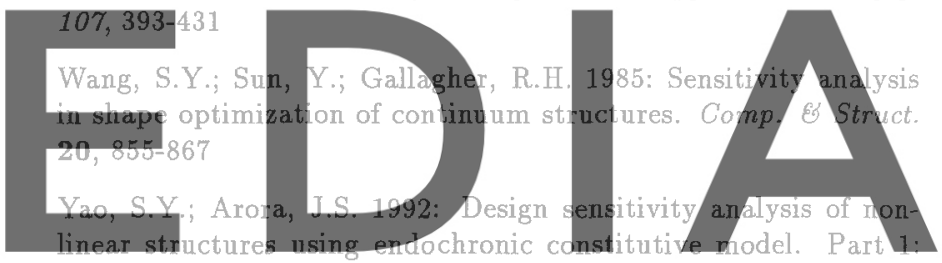

General development. Computational Mech. 10, 39-57

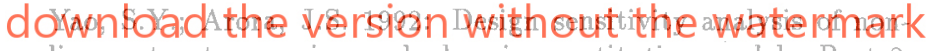
linear structures using endochronic constitutive model. Part 2:

Discretization and applications. Computational Mech. 10, 59-72 